ISSN: 0213-1854

\title{
Metáfora jurídica en la fraseología contrastiva en alemán y en español: el esquema de CAMINO
}

\section{(Legal metaphor in contrastive Phraseology in German and in Spanish: PATH Schema ${ }^{1}$ )}

\author{
LAURA VEgARA FABREgAT/ANA MANSILla PÉREZ \\ lvegara@um.es/anamansi@um.es \\ Universidad de Murcia
}

Fecha de recepción: 10 de febrero de 2017

Fecha de aceptación: 31 de marzo de 2017

Resumen: El lenguaje jurídico está presente en muchos aspectos de nuestra vida cotidiana. Sin duda, se trata de una variedad del lenguaje muy relevante en nuestra sociedad, y muestra de ello, son los diversos trabajos que abundan en este tipo de lenguaje desde la óptica de la semántica, la fraseología o de la lingüística cognitiva, entre otros (Gotti \& Sarcevic, 2006; Méndez-Cendón, 2009). En el presente trabajo nos proponemos estudiar este tipo de lenguaje desde un punto de vista cognitivo a través del esquema de imagen denominado "CAMINO" (Lakoff \& Johnson, 1980) en una serie de unidades fraseológicas del alemán y del inglés. Nuestro objetivo principal no es otro que contribuir con una serie de reflexiones conceptuales y lingüísticas sobre las mencionadas unidades con el fin de arrojar luz sobre la utilidad de la lingüística cognitiva en el campo de la fraseología y la traducción del lenguaje jurídico de los idiomas señalados.

Palabras clave: Fraseología. Metáfora. Esquema de imagen de CAMINO. Lingüística cognitiva. Inglés. Alemán.

Abstract: Legal language is present in many aspects of our daily lives. It is, undoubtedly, a very important type of specialized language. This may be one of the reasons for the proliferation of works studying this variety of language from

\footnotetext{
1 Trabajo enmarcado en el seno del proyecto de investigación FFI2013-45769-P Combinaciones fraseológicas del alemán de estructura [Prep. + Sust.]: patrones sintagmáticos, descripción lexicográfica y correspondencias en español, subvencionado parcialmente con fondos FEDER y dirigido por la Dra. Carmen Mellado Blanco.
} 


\section{Laura Vegara Fabregat/Ana Mansilla PÉREZ}

a semantic, phraseological and translational standpoint, amongst others (Gotti \& Sarcevic, 2006; Méndez-Cendón, 2009). Our goal here is to analyse the mentioned variety of language from a cognitive viewpoint comparing a number of phraseological units belonging to two different linguistic systems, German and English, focusing on the PATH image-schema (Lakoff \& Johnson, 1980). Our main aim is to provide some conceptual and linguistic information, as well as some examples, regarding the aforesaid units in order to shed some light on the areas of phraseology and cognitive linguistics regarding German and English legal language.

Keywords: Phraseology. Metaphor. PATH image-schema. Cognitive linguistics. English. German.

\section{Introducción}

Sin duda, los lenguajes especializados, como el lenguaje periodístico, el turístico o el biomédico, entre otros, han ido cobrando importancia como objeto de estudio en los últimos años. Esto puede deberse, entre otros motivos, a la proliferación de trabajos dedicados al estudio del lenguaje aplicado a parcelas profesionales específicas o a ciertas áreas del saber (Gotti \& Sarcevic, 2006; Méndez-Cendón, 2009). Dichos trabajos a menudo adoptan enfoques concretos como el traductológico o el fraseológico, entre otros, y lo aplican al análisis de los mencionados lenguajes especiales.

En este contexto, nos llama la atención el lenguaje propio del derecho, esto es, el lenguaje jurídico, porque el derecho y la ley inundan nuestras vidas hasta irrumpir en los aspectos más cotidianos como la compra de un billete de tren o el alquiler de un coche, por citar solo dos ejemplos.

Asimismo, es evidente que el lenguaje no se puede desligar de la cognición en general, entre otros motivos, por el hecho de que el hombre tiende a categorizar la realidad de manera innata. El ser humano percibe el mundo de manera estructurada, tal y como señalan diversos expertos (Rosch, 1978: 190). Esta concepción del mundo nos permite interactuar con la compleja realidad que nos rodea, y organizar las entidades y los procesos mentales, entre otras cosas. En consecuencia, creemos que un estudio cognitivo de estas características puede ser interesante y revelador para la fraseología contrastiva y la traducción especializada jurídica, ya que puede proporcionar información relevante sobre el lenguaje, su complejidad, su función y su uso.

Con tal fin en el presente artículo nos proponemos ofrecer una serie de reflexiones cognitivas de tipo cualitativo-descriptivo en torno a una selección de unidades fraseológicas (UF) del ámbito del lenguaje jurídico en el par de 
Metáfora jurídica en la fraseología contrastiva en alemán y en español...

lenguas alemán y inglés. Todo ello desembocará en una serie de conclusiones que, esperamos, arrojen algo de luz en los terrenos y áreas de estudio arriba descritos.

Hemos de indicar que no incluiremos equivalentes en español, ya que la idea principal de nuestro estudio surge de la similitud que, en principio, muestran el inglés y el alemán en muchos aspectos por tener raíz germánica. Asimismo, cabe señalar que en el ámbito jurídico tanto el inglés como el alemán son más prolíficos en metáforas y otras figuras que el español, el cual puede mostrar lenguaje retórico en este ámbito especializado, pero siempre supeditado a un determinado tipo textual y siendo en general menos propenso que el inglés o el alemán (Vegara, 2013: 221-222; Vegara, 2016: 104; Kleinhiespass, 2004; Eckardt, 2002; Jumanca, 2013; Szubert 2015). Efectivamente, algunos autores apuntan que, por ejemplo, el lenguaje administrativo español huye de las imágenes poéticas o literarias, y solo recurre a algunas que están lexicalizadas ya en el uso diario, y a veces a algunas específicas suyas (Calvo, 1980: 212). No cabe duda de que el español jurídico ha sido objeto de diversos trabajos en lo referente a la metáfora (en muchos casos en su vertiente cognitiva pero no siempre) o al lenguaje retórico en general (Hernando, 2003; Gómez, 2005; Chierichetti, 2008, entre otros), pero estos estudios son menos numerosos que los dedicados al lenguaje empleado en sectores como la economía o la publicidad, por citar dos ejemplos.

\section{El lenguaje jurídico y la Lingüística Cognitiva}

Los autores en los que nos inspiraremos parcialmente para la clasificación y el análisis de datos son George Lakoff y Mark Johnson, quienes proponen una perspectiva sobre el lenguaje y la cognición humana que se ha ido desarrollando con el tiempo y que fue revolucionaria y pionera en su momento (Lakoff \& Johnson, 1980). Su teoría se enmarcaría dentro de la vertiente de la Lingüística Cognitiva (LC), la cual estima que el significado lingüístico es enciclopédico y dependiente, esto es, que se basa en nuestra experiencia del mundo y del entorno, así como en otras influencias fuera de nuestro entorno (Lakoff \& Johnson, 1980: 18; 1999: 77 y 90, entre otras). Esta es la tendencia en la que nos hemos centrado, aunque hay diversas ramas dentro de la $\mathrm{LC}^{2}$.

La teoría cognitiva de Lakoff y Johnson (1980) postula, grosso modo, que la metáfora es un recurso cognitivo básico y fundamental (Lakoff \& Johnson, 1980: 3): “[...] pervasive in everyday life, not just in language but in thought and

${ }_{2}^{2}$ Cognitive Grammar, R. Langacker; Grammar Construal, L. Talmy; Frame semantics, C. Fillmore, entre otras. 


\section{Laura Vegara Fabregat/Ana Mansilla PÉREZ}

action". Expresado de manera muy breve, esta perspectiva defiende que concebimos el mundo mediante metáforas y que este tipo de pensamiento es posible gracias a la proyección metafórica, es decir, un proceso mental que toma nociones físicas y/o cotidianas (dominio fuente) y las proyecta sobre ideas o vivencias abstractas (dominio meta) (Lakoff \& Johnson, 1999: 71). Por motivos de espacio, no nos extenderemos en detallar estos postulados, pero remitimos a las obras que los exponen: Lakoff \& Johnson (1980 y 1999) y Johnson (1987).

En esta línea, cabe señalar que existen una serie de trabajos, en los que no abundaremos aquí por razones de brevedad y concisión, que aúnan metáfora (cognitiva) y derecho, entre los cuales destacan: Müller-Dietz (1993), Henly (1987), Kleinhietpass (2004), Dickerson (1996), Benz (2011), Lipshaw (2012) o Jumanca (2013). Estos abordan el estudio de la metáfora en el marco del lenguaje jurídico, en muchos casos desde una perspectiva cognitiva (como es el caso de Jumanca o de Kleinhietpass). No obstante, ninguno de los trabajos citados emplea la combinación lingüística alemán-inglés.

\subsection{El esquema de imagen de CAMINO}

Ciertamente, describir los esquemas de imagen no algo es fácil de lograr en pocas líneas, pero se podrían definir de la siguiente manera: “[...] patrones abstractos recurrentes en nuestro sistema cognitivo, que, a su vez, lo organizan y lo dotan de sentido; proceden de nuestra experiencia corporal [...] así como de nuestra interacción con el entorno" (Vegara, 2013: 177). Existen varios tipos de esquemas, pero en el presente estudio pondremos el punto de mira fundamentalmente en el esquema de CAMINO, gracias al cual las metas y los objetivos pueden concebirse como destinos físicos (Johnson, 1987: 114-115). Este esquema de imagen se encuentra directamente relacionado con otro esquema, el de ORIGEN-RUTA-META (SOURCE-PATH-GOAL) (Lakoff \& Johnson, 1999: 33-34), que se basa en nuestra experiencia corporal del movimiento, pues cada vez que nos movemos, vamos hacia alguna parte. Además, hay un punto de partida y otro de llegada, y pasamos por lugares intermedios (Lakoff, 1987: 275). Teniendo en cuenta lo anterior, Lakoff y Johnson formulan tres componentes esenciales en el esquema CAMINO: un origen, un destino o meta y una dirección por lugares que unen el punto de origen con el de llegada.

Así pues, experiencias que podríamos definir como abstractas, tales como ir a juicio (vor Gericht gehen/to take legal actions), proceder a la aprobación de algo (zur Abstimmung schreiten/to come to the vote), llegar a un acuerdo (zu einer Einigung kommen/to come to an agreement) o evadir la pena de muerte (der Todesstrafe 
Metáfora jurídica en la fraseología contrastiva en alemán y en español...

entgehen/to escape capital punishment) pueden ser representadas como distintas formas de conceptualizar un camino.

A la luz de lo expuesto en estas líneas, en este estudio hemos enfocado el esquema de imagen de CAMINO desde dos ángulos: un camino recto (o convencional) y un camino circular u oblicuo (circunvalación o desvío); es decir, los actos o procesos jurídicos pueden seguir el camino habitual o, por el contrario, seguir rutas alternativas que retroceden, circunvalan el camino o se desvían del mismo. Entrarían entonces en escena los sub-esquemas de CAMINO, como el de DESVIACIÓN, que viene explicitado de forma clara de la siguiente manera: "Si el progreso de una fuerza o entidad es bloqueado por otra fuerza o entidad, una de ellas se verá obligada a desviarse de su ruta inicial" (Peña, 2003: 161).

Cabe aclarar en este punto que, a efectos del presente análisis y con el fin de ser concisos, entendemos que el esquema de CAMINO comprendería todas aquellas expresiones que indican, no solo una trayectoria, línea o camino trazado en sí, sino también el desplazamiento y el movimiento por esa trayectoria (Vegara, 2013: 327), ya sea el movimiento recto, circular o desviado, como indicamos arriba.

Igualmente, y volviendo a la teoría de Lakoff y Johnson, los esquemas de imagen constituyen una de las bases para la formación de las metáforas conceptuales. Del esquema de CAMINO se deriva, por ejemplo, la metáfora conceptual LOS OBJETIVOS SON DESTINOS, como apunta Moreno (2008: 197), entre otros. Por tanto, como se observará más adelante, este esquema hace posible que en nuestra clasificación de datos existan varias categorías (esto es, metáforas conceptuales derivadas) como LLEVAR A CABO UN ACTO/PROCESO JURÍDICO ES LLEGAR AL FINAL DE UN CAMINO, que aglutina metáforas lingüísticas como zu einer Einigung kommen (to come to an agreement) o ein Urteil ergehen (to pass a judgement).

Así pues, las metáforas conceptuales que aparecen en nuestra clasificación y que vamos a desarrollar a continuación son las que siguen:

a. TRAMITAR UN ACTO/PROCESO JURÍDICO ES CAMINAR HACIA ADELANTE

b. LLEVAR A CABO UN ACTO/PROCESO JURÍDICO ES LLEGAR HASTA EL FINAL DE UN CAMINO

c. NO LLEVAR A CABO UN ACTO/PROCESO JURÍDICO ES DESVIARSE DE UN CAMINO/ CIRCUNVALAR UN CAMINO 


\section{LAURA VEgara FABregat/ANA MANSILla PÉREZ}

\section{Metodología de análisis}

En la sección que aquí abrimos procederemos a detallar cuáles han sido nuestros criterios de selección de datos, así como los pasos que hemos seguido para confeccionar la clasificación de los mismos. Seguidamente ofreceremos el análisis cualitativo del corpus.

Siguiendo a Corpas (1996: 52), hemos tenido en cuenta en nuestro corpus todas aquellas expresiones que no constituyen enunciados completos y se componen sobre todo de la estructura verbo + sustantivo y sustantivo + adjetivo. En consecuencia, nos centramos en locuciones verbales y nominales (vor Gericht gehen, gegen jdn. vorgehen; ein gerichtliches Vorgehen, etc.). Tomando como punto de partida este planteamiento, hemos rastreado expresiones en distintos corpus, diccionarios y glosarios electrónicos y en papel tales como el MerriamWebster Online Dictionary o el DUDEN 11, así como en corpus lingüísticos en línea y motores de búsqueda (British National Corpus, Google o Yahoo). Hemos partido siempre del alemán y luego hemos buscado los posibles equivalentes en inglés. En la lengua alemana hemos consultado el ya mencionado DUDEN 11, el Idiomatik Deutsch-Spanisch (2013), el DWDS Online, etc. Para ello hemos buscado verbos (gehen) y sustantivos prototípicos (Weg, Strecke), que aparecen asociados a este esquema en otros trabajos, como el de Moreno (2008) o el de Vegara (2013), los cuales han sido empleados a modo de palabras clave para las búsquedas tanto en alemán como en inglés. Para la búsqueda de equivalentes en inglés hemos empleado fuentes como PONS Online Dictionary o el Beseler Deutsch-English Übersetzer, por citar dos ejemplos. Para elaborar la clasificación de datos nos hemos inspirado principalmente en los postulados básicos de Lakoff y Johnson (1980 y 1999), ya descritos, así como en algunos trabajos, como el de Vegara (2013).

No obstante, nos gustaría resaltar el hecho de que a menudo no se cuenta con corpus fraseológicos actualizados ni con diccionarios totalmente modernizados. Además, estos últimos suelen adolecer de incluir solo las entradas más frecuentes. Para solventar estos problemas, autores como Grefenstette (2002: 201-211) abogan por el uso de la red como corpus o fuente de corpus. Aun así, habría que apuntar que en tales casos sería necesario actuar con cautela en el uso en la red (Mansilla, 2010: 35).

\section{Análisis cualitativo de datos: las expresiones basadas en el esquema de CAMINO}

A continuación, ofrecemos el análisis detallado de los datos, el cual dará paso posteriormente a una serie de conclusiones que darán cuenta de algunas diferencias y similitudes cognitivas entre el alemán y el inglés respecto de ciertos conceptos jurídicos. Por motivos de espacio, no podemos comentar en este 
Metáfora jurídica en la fraseología contrastiva en alemán y en español...

apartado todas y cada una de las expresiones fraseológicas que hemos podido identificar. Por ello, las hemos compilado todas en una tabla (Anexo I) para su consulta.

Recordemos que las expresiones que vamos a analizar son fraseologismos (UF) que responden cognitivamente al esquema de imagen de CAMINO, y que se dividen en las categorías que exponemos seguidamente:

\subsection{TRAMITAR UN ACTO/PROCESO JURÍDICO ES CAMINAR HACLA ADELANTE}

Desde un punto de vista cognitivo, observamos que los procesos o actos jurídicos, normalmente algo abstracto, a menudo se pueden entender como el avance por un camino hacia adelante. En la Tabla 1 a continuación podemos apreciar algunos ejemplos lingüísticos que ilustran esta idea y que comentaremos con detalle más abajo:

\begin{tabular}{|c|c|}
\hline Alemán & Inglés \\
\hline vor Gericht gehen & to take legal action \\
\hline gegen ein Urteil vorgehen & to appeal against a judgement \\
\hline gegen jdn. gerichtlich vorgehen & to bring an action against sh. \\
\hline dem Gesetr folgen & to follow the law \\
\hline die Regel verfolgen & to follow the rule \\
\hline einem Fall nachgehen & to follow up a case \\
\hline ein Recht verfolgen & to pursue a right \\
\hline die Vorschrift befolgen & to follow the rule \\
\hline einen Rechtsweg beschreiten & to go to law/ court/ to take legal action/to \\
& take legal steps \\
\hline
\end{tabular}

Tabla 1

A la hora de elaborar nuestro corpus nos hemos dado cuenta de que los equivalentes $^{3}$ ingleses de los fraseologismos alemanes subyacentes al esquema de CAMINO recurren a veces a una serie de verbos que descansan más bien en la archimetáfora desarrollada por Lakoff y Johnson (1980: 10) LAS IDEAS SON OBJETOS. Muestra de ello es, por ejemplo, el uso del verbo inglés to take (to take legal actions, to take a legal step, etc.), que aparece en la Tabla 1, y que sugiere la manipulación física de un objeto. La expresión inglesa to take legal action se correspondería con rechtliche Schritte einleiten en alemán, y aquella se

3 Empleamos la denominación "equivalente" teniendo en cuenta que en Traducción los equivalentes se suelen valorar en función del contexto y son relativos. 
desprendería cognitivamente en cierto modo de la metáfora conceptual LAS PALABRAS SON OBJETOS y, podríamos decir, también de DEMANDAR ES MANIPULAR UN OBJETO.

Es importante resaltar en este primer grupo de UF que están fundamentadas en verbos que denotan desplazamiento hacia adelante (vorgehen - to go to) o la idea de dirigirse hacia algo (folgen - to follow). El equivalente inglés de vorgehen, to proceed, también aparece en expresiones del ámbito jurídico y descansa, asimismo, en el esquema de CAMINO. No en vano en la etimología de la palabra "proceso", se encuentra la noción de "adelantar, ir delante"4. Un sinónimo en alemán lo hallamos en el sustantivo Verfahren (fahren: marchar, avanzar en un vehículo ${ }^{5}$.

Por otra parte, en el contexto jurídico alemán la UF einen Rechtsweg bescbreiten (to go to law/court) apunta a ser uno de los ejemplos más prototípicos para verbalizar que alguien recurre a los tribunales (esto es, que emprende acciones legales). En este caso, las acciones legales se vinculan al término Schritt (paso) o a sustantivos compuestos del lexema Weg (camino), como se desprende de las UF den Rechtsweg beschreiten y den Klageweg bescbreiten. El verbo beschreiten significa tanto "ir a lo largo de un camino dando pasos" como "introducirse en un camino"6.

El inglés y el alemán parecen compartir el esquema de CAMINO, si nos fijamos en las UF vor Gericht gehen y to go to law/court. Asimismo, resulta curioso observar que en la UF inglesa to go to law se produce una metonimia al sustituir el sitio físico del juzgado (court) por las normas que rigen el derecho o la ley de una nación (law), metonimia que podría enunciarse, en términos generales, como AQUELLO QUE SE IMPARTE /SE HACE CUMPLIR EN UN LUGAR POR EL LUGAR. En cambio, la UF vor Gericht gehen opera de manera opuesta en relación con la metonimia subyacente, esto es, EL LUGAR POR AQUELLO QUE SE IMPARTE/HACE CUMPLIR EN EL LUGAR. Recordemos que en muchos casos la metonimia es la base de la metáfora, pues estos dos fenómenos lingüísticos están íntimamente asociados y suelen darse de manera simultánea (Barcelona, 2000: 53).

4.2. LLEVAR A CABO UN ACTO/ PROCESO JURIDICO ES LLEGAR HASTA EL FINAL DE UN CAMINO

${ }^{4}$ Corominas, J., Breve diccionario etimológico de la lengua castellana. Madrid: Gredos, 1967, p. 476.

${ }^{5}$ Das Digitale Wörterbuch der deutschen Sprache. Disponible en línea en: http://www.dwds.de/?qu=fahren

${ }^{6}$ Das Digitale Wörterbuch der deutschen Sprache. Disponible en línea en: http://www.dwds.de/?qu=beschreiten. 
Metáfora jurídica en la fraseología contrastiva en alemán y en español...

Como comentábamos en la parte dedicada a la fundamentación teórica, un elemento indispensable en la lógica del esquema de CAMINO es, entre otros, un destino o una meta, el propio final del camino (Lakoff \& Johnson 1999: 33 34). Las siguientes UF indican la idea de alcanzar un destino al completar una determinada acción:

\begin{tabular}{|c|c|}
\hline Alemán & Inglés \\
\hline Zu einem Urteil kommen & to arrive at/reach a verdict/judgement \\
\hline Eine Entscheidung ergehen & to pass a resolution \\
\hline Ein Urteil ergehen & to pass a judgement \\
\hline Vorkerungen treffen & to adopt measures \\
\hline Maßnahmen treffen & to take measures \\
\hline Ein Testament vollstrecken & to execute a testament/ a will \\
\hline Ein Urteil vollstrecken & to carry out a sentence \\
\hline Zwangsvollstreckung & foreclosure \\
\hline Testamentvollstrecker & testament executors \\
\hline Vollstreckungsbescheid & enforcement order \\
\hline Die Vollstreckung eines Urteils anordnen & to order for the execution of a sentence \\
\hline
\end{tabular}

Tabla 2

En lo referente a las expresiones en inglés, se aprecia que algunos de los verbos que se asocian con la metáfora conceptual que da nombre a este epígrafe son arrive at y reach (a verdict/judgement); estos llevarían implícitos el hecho de llegar al final de un proceso. En alemán hallamos zu einem Urteil kommen, al principio de la Tabla 2, el cual ilustra la misma idea de llegar a una meta o final. Asimismo, hemos encontrado el verbo ergeben ${ }^{7}$ (ein Urteil ergehen/eine Entscheidung ergehen), cuyo matiz de alcanzar una meta física estaría ciertamente muy lexicalizado y resultaría bastante opaco incluso para el lector nativo alemán.

Una de las muestras que consideramos sugerentes (al menos, parcialmente) en cuanto a la idea de alcanzar la meta o el destino viene representada en alemán por el verbo vollstrecken, que etimológicamente alude a "extender tensando algo sobre una superficie" y cuyo significado translaticio es "ejecutar, llevar a efecto". El inglés, por su parte, se apoya en verbos como to execute o to carry out. Sin embargo, hemos de apuntar que este último se desvía parcialmente del esquema de CAMINO, ya que alude también a la idea de manipular un

${ }_{7}$ Das Digitale Wörterbuch der deutschen Sprache. Disponible en línea en: http://www.dwds.de/?view=1\&qu=ergehen 


\section{LAura Vegara FABregat/AnA MANSILla PÉREZ}

objeto (recordemos la metáfora conceptual ya mencionada LAS IDEAS SON OBJETOS). El verbo inglés to execute, el cual viene etimológicamente del latín ex+sequor (sequor: seguir) ${ }^{8}$, estaría íntimamente ligado, por el matiz etimológico de "seguir", al verbo to follow, y, por tanto, sí podemos hablar de un matiz de desplazamiento, si bien lexicalizado. Este verbo inglés parece encajar mejor conceptualmente en el epígrafe 4.1.

En algunos casos, como muestra la Tabla 2, las expresiones en inglés no indican equivalencia con el alemán en cuanto a la idea de LLEGAR HASTA EL FINAL DE UN CAMINO, pues conceptualmente se ajustarían a otra metáfora o imagen conceptual. Sería, entre otros, el caso de foreclosure o enforcement order, unidades fraseológicas cuya idea subyacente parece ser más bien de tipo espacial (fore $-{ }^{9}$, "in, toward or near the front") o relacionada con la fuerza física (to en-force ${ }^{10}$ ).

Resulta de gran importancia aclarar que en el presente trabajo mostramos metáforas y expresiones con distintos grados de lexicalización, esto es, detectamos algunas UF que pueden resultar más evidentes o transparentes (to follow a case) y otras más opacas (to execute, ergehen). Sin duda, el aspecto de la lexicalización es relevante a la hora de realizar ciertas tareas, como traducir metáforas, tal y como apuntan algunos autores como Samaniego et al. (2005: 66). Sin embargo, no abundaremos en este factor por cuestiones de espacio.

\subsection{NO LLEVAR A CABO UN ACTO/PROCESO JURÍDICO ES DESVIARSE DEL CAMINO/CIRCUNVALAR EL CAMINO}

Cuando determinados asuntos o trámites judiciales y/o jurídicos no siguen su curso habitual hasta el final, el sistema conceptual humano proyecta la idea de tomar un recorrido o un camino inusual (fuera del establecido). Dicha proyección podría aplicarse incluso a la idea de no cumplir la ley en general (transgredir una norma, etc.). A fin de ilustrar con más claridad esta cuestión, veamos los siguientes ejemplos:

\begin{tabular}{|c|c|}
\hline Alemán & Inglés \\
\hline Ein Gesetz umgehen & to evade the law/ to circumvent the law \\
\hline den Vorschriften entgehen & to evade the law \\
\hline eine Klage abweisen & to dismiss / to reject a claim \\
\hline
\end{tabular}

${ }^{8}$ Skeat, W.W., The Concise Dictionary of English Etymology. Hertfordshire: Wordsworth, 2007, p. 425.

${ }_{9}^{9}$ Merriam Webster Online. Disponible en línea en:

http://www.merriam-webster.com/dictionary/fore-

${ }_{10}$ Merriam Webster Online. Disponible en línea en:

http://www.merriam-webster.com/dictionary/enforce 
Metáfora jurídica en la fraseología contrastiva en alemán y en español...

\begin{tabular}{|c|c|}
\hline Der Strafe entgehen & to escape punishment \\
\hline Ein Verbot übertreten & to contravene a ban \\
\hline Ein Gesetz überschreiten & to overstep the law \\
\hline eine Klage zurückweisen & to reverse a judgement \\
\hline
\end{tabular}

Tabla 3

Algunos de los fraseologismos recogidos en la Tabla 3 nos transmiten la idea de que cuando no seguimos las normas o leyes vigentes las "rodeamos" (ein Gesetr umgehen) o incluso pasamos por encima de ellas, en ocasiones "pisándolas", (ein Gesetz überschreiten). Encontramos en inglés expresiones que pueden ser casi totalmente convergentes con las UF en alemán que hemos hallado, como es el caso de to overstep the law/ein Gesetz überschreiten. Un caso que nos parece parcialmente divergente (porque recae en la idea de CAMINO pero no de la misma manera) es el de ein Verbot übertreten/ to contravene a ban, el cual comentamos con detalle más abajo.

La metáfora conceptual que da nombre a la presente categoría incluye también la idea de CIRCUNVALAR UN CAMINO, la cual sería responsable de las siguientes UF que hemos encontrado: ein Gesetz umgehen, Steuer umgehen, die Vorschriften umgehen, etc. (Anexo I). Como se aprecia en la Tabla 3, a diferencia del alemán, que describe la imagen del círculo (o, al menos, el acto de rodear un camino a través del significado literal de umgehen), el inglés parece desmarcarse de dicha imagen en ocasiones para centrarse más bien en el significado metafórico de salirse de un camino, escaparse de él. Esto implica un movimiento de dentro hacia fuera como se observa, por ejemplo, en fraseologismos como to evade the law, to evade taxes (del latín evadere: to go, walk) ${ }^{11}$, etc. No obstante, hemos de recalcar la existencia del verbo de procedencia latina to circumvent en inglés, denotativo de movimiento circular, el cual se observa en algunas UF como to circumvent the law (Tabla 3).

Finalmente, recogemos UF en inglés que contienen verbos como dismiss, contravene y reverse. En el caso del primer verbo, podemos considerar que el matiz

11 Merriam Webster Online Dictionary. Disponible en línea en:

http://www.merriam-webster.com/dictionary/evade 


\section{LAUra VEgara FABregat/ANA MANSILla PÉREZ}

de movimiento está más bien lexicalizado, aunque etimológicamente dismiss posee una raíz latina que significa "send, let go" 12 . Con contravene sucede algo similar, del latín contra + venire ${ }^{13}$, parece indicar movimiento en contra de algo o alguien. Con reverse apreciamos más claramente el matiz de movimiento hacia atrás. Su significado es bastante transparente. En estos casos la noción de desviarse o circunvalar un camino no está presente o, al menos, no de manera tan clara como sucede en alemán.

\section{Conclusiones}

En el presente estudio nuestro objetivo principal ha sido extraer y analizar de manera cualitativa una serie de unidades fraseológicas del lenguaje jurídico en alemán y en inglés. El eje de dicho análisis lo ha constituido el esquema de CAMINO. Antes de exponer nuestras reflexiones finales, nos gustaría destacar una dificultad añadida de aplicar el enfoque cognitivo a las expresiones jurídicas que hemos estudiado: la naturaleza polisémica de las unidades fraseológicas complica en gran medida la elaboración de metáforas conceptuales concretas e infalibles. Por tanto, las unidades que hemos asociado a cada metáfora podrían responder a más de una e incluso pertenecer a otras metáforas conceptuales que no hemos recogido.

Por una parte, hemos observado que, dentro de la primera categoría analizada, algunas de las expresiones alemanas subyacentes al esquema de CAMINO descansan en inglés en la metáfora denominada LAS PALABRAS SON OBJETOS (relacionada a su vez con LAS IDEAS SON OBJETOS). A pesar de ello, varias de las unidades estudiadas indican que el alemán y el inglés sí comparten el esquema de CAMINO en expresiones del tipo vor Gericht gehen/ to go to court.

Por otra, cabe señalar que la metáfora conceptual IDEAS SON OBJETOS vuelve a aparecer en relación con la segunda categoría, la metáfora LLEVAR A CABO UN ACTO/PROCESO JURÍDICO ES LLEGAR HASTA EL FINAL DE UN CAMINO, pues vemos que, nuevamente, varias expresiones inglesas poseen como base cognitiva dicha metáfora (to take measures, to carry out), y no el esquema de CAMINO.

Asimismo, el alemán y el inglés convergen en muchos casos en cuanto a la última metáfora, NO LLEVAR A CABO UN ACTO/PROCESO JURÍDICO ES DESVIARSE DEL CAMINO/CIRCUNVALAR UN CAMINO. Sin

12 Online Etymology Dictionary. Disponible en línea en: http://www.etymonline.com/index.php?term=dismiss .

13 Online Etymology Dictionary. Disponible en línea en:

http:/ /www.etymonline.com/index.php?allowed_in_frame $=0 \&$ search $=$ contravene . 
Metáfora jurídica en la fraseología contrastiva en alemán y en español...

embargo, en lo relativo a la imagen de "circunvalar", se aprecia que mientras que el alemán recurre a la imagen del círculo el inglés parece centrarse en la de escapar o salirse del camino (to evade the law, to evade taxes).

En definitiva, los dos grupos de UF jurídicas estudiados parecen compartir el esquema de CAMINO a tenor de muchos de los ejemplos analizados, aunque también presentan divergencias conceptuales. No obstante, dado que nuestro estudio es de naturaleza eminentemente cualitativa, no podemos hacer generalizaciones amplias.

El esquema de CAMINO podría sin duda ser explotado cuantitativamente de manera exhaustiva en el ámbito descrito, pues parece que podría ser mucho más prolífico. Un estudio cuantitativo alemán-inglés de este esquema en el área del derecho podría revelar pautas recurrentes y otros datos muy interesantes, así como de gran utilidad en el campo de la fraseología, la traducción y/o la lingüística cognitiva.

\section{Referencias bibliográficas}

Álvarez Calleja, M. Ángeles, "El Lenguaje Económico como Fuente de Imágenes Metafóricas". En: The Golden Bowl, de Henry James. IV Encuentros Complutenses en torno a la traducción, Margit Raders \& Rafael MartínGaitero (eds.). Madrid: Editorial Complutense, 1994, 467-476.

BARCELONA, Antonio (ed.), "On the plausibility of claiming a metonymic motivation for conceptual metaphor". En: Metaphor and Metonymy at the Crossroads, (ed.) Antonio Barcelona. Berlín: Mouton de Gruyter, 2003, 31-58.

BENZ, Wolfgang, “'Heimat' als Metapher im Diskurs der völkerischen Rechten". En: Ausschluss und Feindschaft: Studien zu Antisemitismus und Rechtsextremismus, (ed.) Michael Kohlstruck y Andreas Klämer. Berlín: Metropol, 2011, 124-134.

Beseler DeustSCh-ENGlish ÜBERSETZER. Disponible en línea en: http://langua.de/eng/Beseler. [Fecha de consulta: 20 de octubre de 2014]

BRITISH NATIONAL CORPUS. Disponible en línea en: http://www.natcorp.ox.ac.uk/. [Fecha de consulta: 20 de octubre de 2014]

COROMINAS, Joan, Breve diccionario etimológico de la lengua castellana. Madrid: Gredos, 1967.

CALVO, Luciana, Introducción al estudio del lenguaje administrativo. Madrid: Gredos, 1980.

CORPAS, Gloria, Manual de fraseologia española. Madrid: Gredos, 1996.

DAS DIGITALE WÖRTERBUCH DER DEUTSCHEN SPRACHE. Disponible en línea en: http://www.dwds.de/. [Fecha de consulta: 20 de noviembre de 2014] 


\section{LAURA VEgara FABregat/ANA MANSILla PÉREZ}

CHIERICHETTI, Luisa, "La comunicación especializada de ámbito jurídico: una reflexión sobre las metáforas en los ordenamientos penales español e italiano”. En: Navarro, Carmen / Rodríguez Abella, Rosa M., Dalle Pezze, Francesco / Miotti, Renzo (eds), La comunicación especializada. Bern: Peter Lang, 2008, 203-217.

DiCKERSON, Donna, “Freedom of expression' and Cultural Meaning: An Analysis of Metaphors in Selected Supreme Court Texts". En: Journal of Communications Law and Policy, 1, 1996, 367-395.

DUDEN ONLINE. Disponible en línea en: http://www.duden.de/. [Fecha de consulta: 20 de noviembre 2015]

ECKARDT, Birgit, "Metaphorik in der rechtswissenschaftlichen Fachkommunikation”. En: Fachsprache/International Journal of LSP, 24, 3-4, 2002, 140-148.

FILLMORE, Charles, "Frame semantics and the nature of language". En: Annals of the New York Academy of Sciences: Conference on the Origin and Development of Language and Speech, 280, 1976, 20-32.

GÓMEZ BORRÁs, Marta, “La nueva retórica y el nuevo lenguaje jurídico”. En: RJN, 39, 2005, 97-138.

GOTTI, Maurizio y SARCEVIC, Susan, Insights into Specialized Translation. Berna: Peter Lang, 2006.

GREFENSTETTE, Gregory, "The WWW as a Resource for Lexicography". En: Lexicography and Natural Language Processing: A Festschrift in Honour of Beryl Atkins, (ed.) Marie Hélène Corréard. Gotemburgo: Euralex, 2002, 199-215.

Henly, Burr, "Penumbra: Roots of Legal Metaphor". Hastings Constitutional Law Quaterly, 15/81, 1987, 81-100.

Hernando CuAdrado, Luis Alberto, El lenguaje jurídico. Madrid: Verbum S.L., 2003.

JoHnson, Mark, The Body in the Mind: the Bodily Basis of Meaning, Imagination and Reason. Chicago: University of Chicago Press, 1987.

JUMANCA, Romanița, "Types of Metaphors in the English Legal Discourse". En: Romanian Journal of English Studies, 9, 1, 2013, 366-372.

KLEINHIETPASS, Cordula, Metaphern der Rechtssprache und ihre Verwendung für Visualisierungen. Berlín: Tenea Verlag, 2005.

LAKOFF, George y JOHNSON, Mark, Metaphors we live by. Chicago: University of Chicago Press, 1980.

LAKOFF, George, Women, Fire and Dangerous Things. Chicago: University of Chicago Press, 1987.

LAKOFF, George y JOHnson, Mark, Philosophy in the Flesh: The Embodied Mind and Its Challenge to Western Thought. Nueva York: Basic Books, 1999. 
Metáfora jurídica en la fraseología contrastiva en alemán y en español...

LANGACKER, Ronald Wayne, Foundations of Cognitive Grammar. Volumen 1, Theoretical Prerequisites. Stanford: Stanford University Press, 1987.

LipSHAw, Jeffrey, "Metaphors, Models, and Meaning in Contract Law". En: Penn State Law Review, 116/4, 2012, 987-1042.

MANSILLA, Ana, "La ordenación onomasiológica del material fraseológico". En: La fraseografía del S. XXI. Nuevas perspectivas para el español y el alemán, (ed.) Carmen Mellado, Patricia Buján, Claudia Herrero, Nely Iglesias y Ana Mansilla. Berlín: Frank \& Timme, 2010, 27-37.

Mendez-Cendon, Beatriz, "Combinatorial Patterns in Medical Case Reports: An English-Spanish Contrastive Analysis". En: Journal of Specialized Translation, 11, 2009, 169-190.

MERRIAM-WEBSTER ONLINE: Dictionary and Thesaurus. Disponible en línea en: http://www.merriam-webster.com/dictionary/burden. [Fecha de consulta: 10 de octubre 2016]

Moreno, María Ángeles, La metáfora conceptual y el lenguaje político periodístico: configuración, interacciones y niveles de descripción, tesis doctoral. La Rioja: Universidad de la Rioja, 2004.

MÜLlER-DiETZ, Heinz, "Die soziale Wahrnehmung von Kriminalität". En: Neue Zeitscbrift der Kriminalität, 13, 1993, 57-65.

OnLINE ETYMOLOGY Dictionary. Disponible en línea en: http://www.etymonline.com/index.php?term=dismiss. [Fecha de consulta: 4 de febrero de 2016]

PEÑA, Sandra, Topology and Cognition: What Image-Schemas Reveal about the Metaphorical Language of Emotions. LINCOM Studies in Cognitive Linguistics, 2003.

PONS ONLINE DiCTIONARY. Disponible en línea en: http://en.pons.com/translate?q=klagenwege\&l=deen\&in=\&lf=de. $\quad[$ Fecha de consulta: 20 de octubre de 2014]

Rosch, Eleanor, "Principles of Categorization". En: Concepts: Core Readings, (ed.) Eric Margolis y Stephen Laurence. Massachusetts: MIT, 1978, 189-206.

SAMANIEGO, Eva et al., "Translations we live by: the impact of metaphor translation on target systems". En: Lengua y Sociedad: Investigaciones recientes en Lingüistica Aplicada, (ed.) Pedro Fuertes. Valladolid: Universidad de Valladolid, 2005, 61-81.

SZUBERT, Rafal, "Metaphor der Person im juristischen Diskurs". En: Glottodidactica XLII/2, 2015, 101-118.

TALMY, Leonard, "Lexicalisation patterns". En: Language typology and syntactic description. Volume III. Grammatical categories and the lexicon, (ed.) Timothy Shopen. Cambridge: Cambridge University Press, 1985, 57-149. 
VEGARA, Laura, Las metáforas en los textos jurídicos y su traducción al castellano: las resoluciones del Tribunal Supremo de los EEUU. Tesis Doctoral. Alicante: Universidad de Alicante, 2013.

VEGARA, Laura, "El estudio de una opinión estadounidense desde la dimensión del género y su utilidad para la traducción jurídica". En: Hikma. Revista de Traducción, 15, 2016, 95-116.

\section{ANEXO I}

\subsection{TRAMITAR UN ACTO/PROCESO JURÍDICO ES CAMINAR HACIA ADELANTE}

Ein Verbrechen begehen-to commit a crime

Eine Rechtsverletzung begehen-to commit the infringement of a right

Einen Mord begehen- to commit a murder

Einen Rechtsbruch begehen-to commit a breach of the law

Einen Gesetzesverstoß begehen-to commit an infringement of the law

Eine Straftat begehen-to commit a crime / an offence

Selbstmord begehen-to commit suicide

Verrat begehen-to commit treason

Den Rechtsweg beschreiten- to go to law/ to take legal action

Rechtliche Schritte einleiten-take legal action

Ein Rechtsweg zulässig sein- admissible legal action

In die Berufung gehen-to appeal/to file an appeal

Vor Gericht gehen- to take legal action/ to litigate

Gegen ein Urteil vorgehen- to appeal against a judgement

Gerichtliches Vorgehen-legal proceeding

Nach den Vorschriften vorgehen - to adhere to the regulations

Nach einem Verfahren vorgehen - to observe a (legal) procedure

Nach einer Maxime vorgehen - to act on a maxim

Gegen ein Urteil vorgehen- appeal against a judgement

Gegen jdn. gerichtlich vorgehen- to bring an action against sb.

Gegen etw. resolut vorgehen-to act firmly against something

Umlautes Vorgehen- corrupt practices

Dem Gesetz folgen-to follow the law

Gesetze befolgen-to follow the laws

Einen Befehl befolgen-to follow an order

Das Gebot befolgen-to follow a rule/regulation 
Metáfora jurídica en la fraseología contrastiva en alemán y en español...

\begin{tabular}{|l|}
\hline Ein Verfahren laufen-to run a process \\
\hline Ein Prozess laufen- to run legal proceedings \\
\hline Die Regel verfolgen- to follow the rule \\
\hline Einem Fall nachgehen- to follow up a case \\
\hline Ein Recht verfolgen- to pursue a right \\
\hline Strafrechtlich verfolgen-to prosecute sb \\
\hline Die Vorschrift befolgen- to follow the rule \\
\hline Einen Rechtsweg beschreiten- to go to law/to take legal steps \\
\hline Rechtsmittelverfahren - appeal procedure \\
\hline Revisionsverfahren - appeal proceedings \\
\hline Eine Verfahrensordnung- rules of procedure \\
\hline Gesetzgebungsverfahren -legislative procedure \\
\hline Beschlussfassungsverfahren -decision making procedure \\
\hline Vermittlungsverfahren -joint committee procedure \\
\hline Beratungsverfahren - consultation procedure \\
\hline Ermittlungsverfahren- premilinary proceedings \\
\hline Ein Berufungsverfahren- appellate procedure \\
\hline Einspruchsverfahren- opposition procedure \\
\hline Strafverfahren- criminal procedure \\
\hline Gerichtsverfahren-legal court proceedings \\
\hline Eilverfahren- accelerated proceedings \\
\hline Schnellverfahren- speedy trial \\
\hline Rechtsstaatliches Verfahren- rule of law procedure \\
\hline Ein Ausgleichsverfahren- composition proceedings \\
\hline Beschleunigtes Verfahren einführen- to start accelerated proceedings \\
\hline 4.2. LLEVAR A CABO UN ACTO/PROCESO JURÍDICO ES LLEGAR \\
\hline HASTA EL FINAL DE UN CAMINO \\
\hline Ein Urteil vollziehen- to enforce a judgement \\
\hline Zur Abstimmung kommen-to come to the vote \\
\hline In Kraft treten- to come/enter into force \\
\hline Zu einem Urteil kommen- to arrive at/reach a verdict/judgement \\
\hline Eine Entscheidung ergehen- to pass a resolution \\
\hline Ein Urteil ergehen- to pass a judgement \\
\hline Vorkerungen treffen- to adopt measures \\
\hline Maßnahmen treffen- to take measures \\
\hline Ein Testament vollstrecken- to execute a testament/a will \\
\hline Ein Urteil vollstrecken- to carry out a sentence \\
\hline Testamentvollstrecker- testament executors \\
\hline
\end{tabular}




\begin{tabular}{|l|}
\hline Vollstreckungsbescheid- enforcement order \\
\hline $\begin{array}{l}\text { Die Vollstreckung eines Urteils anordnen- to order for the execution of a } \\
\text { sentence }\end{array}$ \\
\hline Zu einem Einverständnis gelangen- to reach an approval \\
\hline Zu einem Urteil gelangen- to reach a judgement \\
\hline Einstimmigkeit erreichen- to come to a unanimous agreement \\
\hline Eine Resolution passieren - to pass a resolution \\
\hline Ein Prozess ist erfolgt- to hold/to have a process \\
\hline Eine Strafverfolgung erfolgen- to hold a criminal prosecution \\
\hline Zu einer einvernehmlichen Einigung gelangen- to come to a mutual agreement \\
\hline $\begin{array}{l}\text { Zu einer Einigung kommen / zur Übereinstimmung kommen- to come to an } \\
\text { agreement }\end{array}$ \\
\hline $\begin{array}{l}\text { 4.3. NO LLEVAR A CABO UN ACTO/PROCESO JURÍDICO ES } \\
\text { DESVIARSE DEL CAMINO/CIRCUNVALAR EL CAMINO }\end{array}$ \\
\hline Den Vorschriften entgehen- to evade/avoid rules/regulations \\
\hline Steuer entgehen - to evade taxes \\
\hline Dem Urteil entgehen - to evade/escape a judgement/sentence \\
\hline Der Todesstrafe entgehen- to escape capital punishment \\
\hline Verpflichtungen entgehen- to avoid obligations \\
\hline Der Klage entgehen -to avoid a lawsuit \\
\hline Der Strafe entgehen- to escape punishment \\
\hline Einen Fall abweisen- to dismiss a case \\
\hline Eine Berufung abweisen- to dismiss an appeal \\
\hline Eine Klage abweisen- to dismiss a case/a suit \\
\hline Ein Gesetz umgehen-to circumvent the law/to evade the law \\
\hline Bestimmungen umgehen - to avoid/to go around regulations \\
\hline Steuern umgehen- to evade taxes \\
\hline Die Vorschriften umgehen-to bend the rules \\
\hline Verpflichtungen umgehen- to avoid duties/obligations \\
\hline Ein Verbot übertreten- to contravene a ban \\
\hline Eine Regel übertreten - to contravene/infringe a rule \\
\hline Ein Gesetz überschreiten- to overstep the law \\
\hline Eine Klage zurückweisen- to reverse a judgement \\
\hline Statuten übertreten - to contravene/violate statutes \\
\hline Bestimmungen übertreten - to contravene/violate regulations \\
\hline Gesetze übertreten - to contravene/violate the laws \\
\hline Vorschriften übertreten - to violate/break the rules/regulations \\
\hline Kriminelles Vergehen - criminal offence \\
\hline
\end{tabular}


Metáfora jurídica en la fraseología contrastiva en alemán y en español...

\begin{tabular}{|l|}
\hline Ein Vergehen verüben - to commit/carry out an offence \\
\hline Einer Verurteilung entkommen- to escape conviction \\
\hline Einem Gesetz entkommen- to escape the law \\
\hline Bürgschaftsfähiges Vergehen - bailable offence \\
\hline Geringfügiges Vergehen - minor misdemeanour \\
\hline Vergehen im Amt - malfeasance in office \\
\hline Das Verfahren verkürzen - to abbreviate proceedings \\
\hline
\end{tabular}

